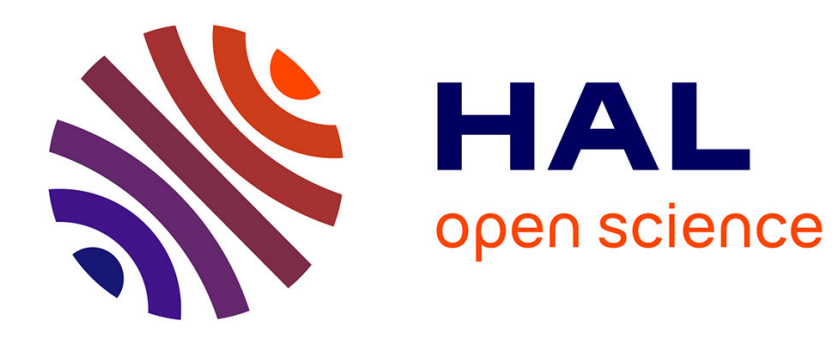

\title{
La pyramide de Kheops et quelques équations du quatrième degré
}

Aimé Lachal

\section{To cite this version:}

Aimé Lachal. La pyramide de Kheops et quelques équations du quatrième degré. Quadrature, 2008, 69, pp.36-47. 10.1051/quadrature:2008004 . hal-01362047

\section{HAL Id: hal-01362047 \\ https://hal.science/hal-01362047}

Submitted on 8 Sep 2016

HAL is a multi-disciplinary open access archive for the deposit and dissemination of scientific research documents, whether they are published or not. The documents may come from teaching and research institutions in France or abroad, or from public or private research centers.
L'archive ouverte pluridisciplinaire HAL, est destinée au dépôt et à la diffusion de documents scientifiques de niveau recherche, publiés ou non, émanant des établissements d'enseignement et de recherche français ou étrangers, des laboratoires publics ou privés. 


\section{La pyramide de Kheops et quelques équations du quatrième degré}

par Aimé Lachal*

\section{Prologue}

Dans un article publié dans l'édition française de la revue australienne Nexus [2], James Colmer présentait une hypothèse originale sur la fonction de la pyramide de Kheops et sur l'existence présumée d'un ensemble de galeries et chambres cachées, symétriques à celles connues. La démarche de J. Colmer, dans sa recherche, passait par un tracé géométrique impliquant une certaine valeur de l'angle formé par la face de la pyramide avec sa base horizontale et la triple intersection d'une circonférence avec deux segments de droites spécifiques.

André Dufour, traducteur pour Nexus France et architecte de métier, ayant eu la charge de traduire cet article, s'aperçut après avoir refait sur ordinateur le tracé géométrique de J. Colmer que ce dernier était faux. Cela n'enlevait rien à l'intérêt de l'hypothèse de J. Colmer, objet principal de l'article, mais posait un problème intéressant de géométrie. Après en avoir fait une note de traducteur publiée simultanément avec l'article traduit de J. Colmer, plusieurs lecteurs, F. De Ligt, J.-F. Pioche et l'auteur du présent article, réagirent au problème en fournissant des valeurs exactes pour l'angle mis en cause par le biais de diverses méthodes calculatoires. Les calculs de F. De Ligt et J.-F. Pioche ont été partiellement rassemblés et commentés par A. Dufour et publiés dans une courte note [3].

Par une démarche empirique, A. Dufour tenta une construction graphique à l'aide d'un logiciel de dessin vectoriel afin d'obtenir la position du sommet de la pyramide requise par la théorie de J. Colmer. Cette approche, qu'il a soumise à l'auteur, repose sur la

\footnotetext{
* Institut National des Sciences Appliquées de Lyon, Pôle de Mathématiques, Bâtiment Léonard de Vinci, 20 avenue Albert Einstein, 69621 Villeurbanne Cedex, France; aime.lachal@insa-lyon.fr
}

construction d'une courbe en polyligne lissée dont l'intersection avec une droite adéquate fournit avec une excellente précision la position du sommet recherché. Il apparaîtra ultérieurement qu'en fait deux sommets conviendront. La figure 19 (qui est l'un des deux graphiques principaux de cet article) correspondant au sommet le plus bas est, à une symétrie près, le tracé initialement proposé par A. Dufour. En adaptant cette construction au cas du sommet le plus élevé, on obtient dans le même esprit le deuxième graphique principal de ce travail, à savoir la figure 20 .

Par une approche analytique, l'auteur prouvera alors que la construction d'A. Dufour est parfaitement exacte. Il est intéressant de noter que, bien au-delà d'un exercice de géométrie en apparence élémentaire, ce problème suscitera en fait une analyse mathématique particulièrement riche touchant à des domaines diversifiés.

Tous les éléments des diverses correspondances entre A. Dufour, F. De Ligt, J.-F. Pioche et l'auteur sont rassemblés dans le présent article.

\section{Description du problème}

On se donne un rectangle dont la base est le double de la hauteur, un triangle isocèle dont la base coïncide avec celle du rectangle et un cercle de rayon le quart de la base du rectangle, tangent à un côté vertical du rectangle et dont un diamètre est porté par un côté latéral du triangle (voir figure 1).

Le problème évoqué dans la section I consiste à rechercher une position pour le sommet du triangle qui produise une triple intersection entre une diagonale du rectangle, le cercle et la perpendiculaire au diamètre du cercle porté par le côté latéral mentionné du triangle (voir figure 1). Nous verrons qu'il y a deux solutions possibles à ce problème (voir figures 2 et 3 ). 


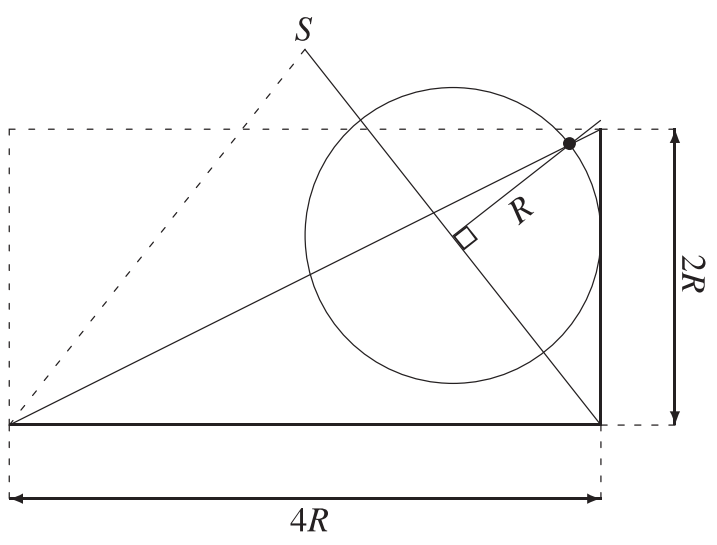

Figure 1. Données du problème.

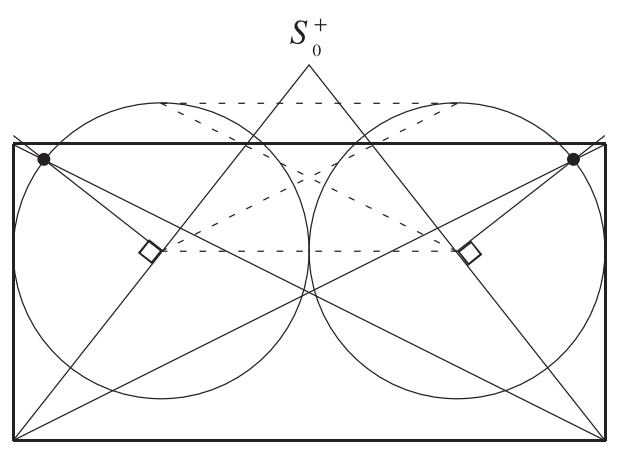

Figure 2. La pyramide de Kheops (1).

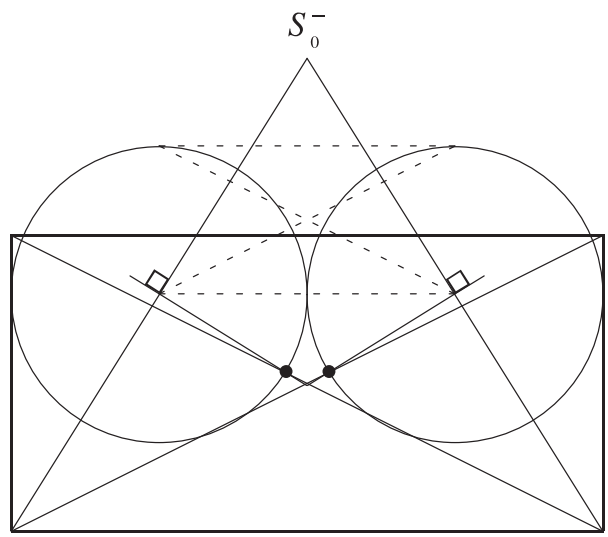

Figure 3. La pyramide de Kheops (2).

Nous avons ébauché en pointillés sur les figures 2 et 3 l'« étoile infinie » (de sommet $S_{0}^{+}$ou $\left.S_{0}^{-}\right)$mentionnée par J. Colmer.

Dans ce travail, nous adopterons plusieurs approches : trigonométrique (section III), analytique (section IV), puis algébrique (section V). Ces diverses approches conduisent toutes à la résolution d'équations du quatrième degré dont les solutions ne sont pas constructibles à la règle et au compas. Malgré l'impossibilité d'une telle construction, nous proposerons néanmoins une construction géométrique originale qui fera l'objet de la section VI.

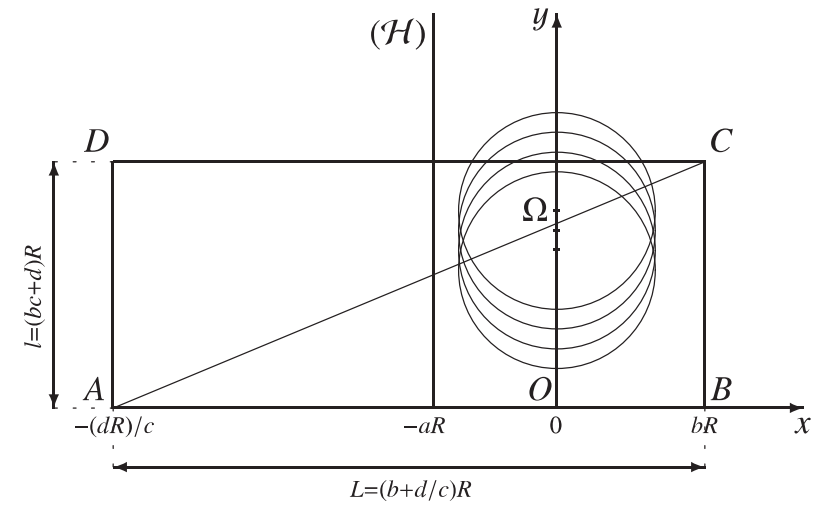

Figure 4. Notations.

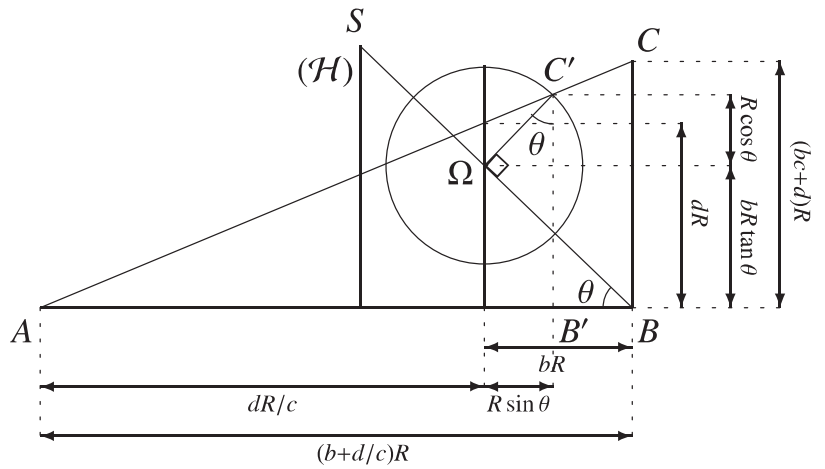

Figure 5. Égalité des pentes dans le triangle $(A B C)(1)$.

On traite le problème dans un cadre plus général pour lequel les dimensions des objets considérés sont quelconques. Les données et notations sont alors les suivantes (voir figure 4).

Soit $(A B C D)$ un rectangle de longueur $A B=L$ et de hauteur $B C=l$. On trace la diagonale $(A C)$, on place une origine $O$ sur la base $(A B)$. L'axe $(A B)$ est choisi comme axe des abscisses et sa perpendiculaire en $O$ comme celui des ordonnées. On considère ensuite un cercle mobile de rayon $R$ dont le centre $\Omega$ coulisse le long de l'axe des ordonnées. Le point $B$ est situé à une distance $b R>0$ de $O$ et la diagonale $(A C)$ a pour équation $y=c x+d R$. On trace enfin une hauteur $(\mathcal{H})$ à une distance $-a R<0$ de $O$. On suppose $a, b \geqslant 1$.

Le cas de la pyramide de Kheops correspond aux valeurs $a=b=1, c=1 / 2$ et $d=3 / 2$.

\section{Résolution trigonométrique}

On cherche les angles $\theta=\widehat{A B \Omega} \in] 0, \pi / 2[$ pour lesquels la perpendiculaire à $(\Omega B)$ issue de $\Omega$ coupe simultanément le cercle $C(\Omega, R)$ et la diagonale $(A C)$ en un point que l'on notera $C^{\prime}$. Les sommets $S$ recherchés seront ensuite obtenus comme intersection de $(\Omega B)$ et de $(\mathcal{H})$. Notons que l'on retrouve l'angle $\theta$

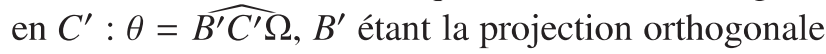




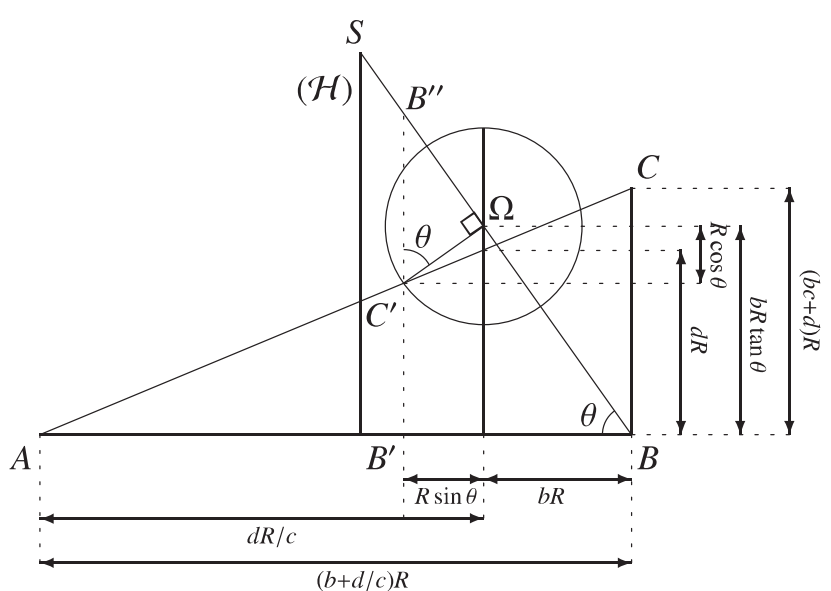

Figure 6. Égalité des pentes dans le triangle $(A B C)(2)$.

de $C^{\prime}$ sur la base $(A B)$ (figure 5) ou $\theta=\widehat{B^{\prime \prime} C^{\prime}} \Omega, B^{\prime \prime}$ étant la projection de $C^{\prime}$ sur la droite $(\Omega B)$ parallèlement à $(\mathcal{H})$ (figure 6).

En égalant les deux expressions $B C / A B$ et $B^{\prime} C^{\prime} / A B^{\prime}$ de la pente $c=l / L$ de la diagonale $(A C)$, on trouve, à l'aide des longueurs indiquées sur la figure 5, la relation $\cos \theta+b \tan \theta=c\left(\frac{d}{c}+\sin \theta\right)$ qui donne $\cos ^{2} \theta+b \sin \theta=c \cos \theta \sin \theta+d \cos \theta$. On peut écrire cette équation sous deux formes :

$$
\cos ^{2} \theta-d \cos \theta=\sin \theta(c \cos \theta-b)
$$

ou

$$
1+b \sin \theta-\sin ^{2} \theta=\cos \theta(c \sin \theta+d) .
$$

La première équation implique

$$
\left(\cos ^{2} \theta-d \cos \theta\right)^{2}=\left(1-\cos ^{2} \theta\right)(c \cos \theta-b)^{2},
$$

d'où l'équation de degré 4 satisfaite par $\cos \theta$ :

$$
\begin{aligned}
& \left(c^{2}+1\right) \cos ^{4} \theta-2(b c+d) \cos ^{3} \theta \\
& \quad+\left(b^{2}-c^{2}+d^{2}\right) \cos ^{2} \theta+2 b c \cos \theta-b^{2}=0 .
\end{aligned}
$$

La deuxième équation implique

$$
\left(1+b \sin \theta-\sin ^{2} \theta\right)^{2}=\left(1-\sin ^{2} \theta\right)(c \sin \theta+d)^{2},
$$

d'où l'équation de degré 4 satisfaite par $\sin \theta$ :

$$
\begin{aligned}
& \left(c^{2}+1\right) \sin ^{4} \theta-2(b-c d) \sin ^{3} \theta+\left(b^{2}-c^{2}\right. \\
& \left.\quad+d^{2}-2\right) \sin ^{2} \theta+2(b-c d) \sin \theta+\left(1-d^{2}\right)=0 .
\end{aligned}
$$

De manière analogue, la situation décrite par la figure 6 conduit à la relation $b \tan \theta-\cos \theta=c\left(\frac{d}{c}-\sin \theta\right)$. Cela revient formellement à changer $b$ et $d$ en leurs opposés par rapport aux équations précédentes et l'on obtient

$$
\begin{aligned}
& \left(c^{2}+1\right) \cos ^{4} \theta+2(b c+d) \cos ^{3} \theta \\
& \quad+\left(b^{2}-c^{2}+d^{2}\right) \cos ^{2} \theta-2 b c \cos \theta-b^{2}=0
\end{aligned}
$$

et

$$
\begin{aligned}
& \left(c^{2}+1\right) \sin ^{4} \theta+2(b-c d) \sin ^{3} \theta+\left(b^{2}-c^{2}\right. \\
& \left.\quad+d^{2}-2\right) \sin ^{2} \theta-2(b-c d) \sin \theta+\left(1-d^{2}\right)=0 .
\end{aligned}
$$

Enfin, une fois les angles $\theta$ trouvés, les hauteurs de la pyramide répondant au problème initialement posé s'obtiennent par les ordonnées des sommets $S$ :

$$
y_{S}=(a+b) R \tan \theta .
$$

Dans l'exemple de la pyramide de Kheops, les équations précédentes s'écrivent respectivement

$$
5 \cos ^{4} \theta \mp 16 \cos ^{3} \theta+12 \cos ^{2} \theta \pm 4 \cos \theta-4=0,
$$

et

$$
5 \sin ^{4} \theta \mp 2 \sin ^{3} \theta+4 \sin ^{2} \theta \pm 2 \sin \theta-5=0 .
$$

Maple fournit les valeurs numériques

$\cos \theta_{0}^{+} \approx 0.6164792130$ et $\sin \theta_{0}^{+} \approx 0.7873711831$, $\cos \theta_{0}^{-} \approx 0.5289114551$ et $\sin \theta_{0}^{-} \approx 0.8486770131$

qui donnent les angles

$$
\begin{aligned}
& \theta_{0}^{+} \approx 0.906533065 \mathrm{rd} \approx 51^{\circ} 56^{\prime} 26^{\prime \prime}, \\
& \theta_{0}^{-} \approx 1.013478912 \mathrm{rd} \approx 58^{\circ} 04^{\prime} 05^{\prime \prime} .
\end{aligned}
$$

Les hauteurs respectives de chacune des deux pyramides correspondantes (de sommets $S_{0}^{+}$et $S_{0}^{-}$) valent finalement, grâce à $y_{S}=2 R \tan \theta$,

$$
y_{s_{0}^{+}} \approx 2.554412758 R \text { et } y_{s_{0}^{-}} \approx 3.209145898 R \text {. }
$$

\section{Résolution analytique}

Plusieurs méthodes analytiques reposant sur des équations de droite et de cercle sont possibles. Nous en proposons deux ci-après.

\section{IV.1 Première méthode}

Le cercle $C(\Omega, R)$ coulisse le long de l'axe des ordonnées et la droite $(\Omega B)$ pivote autour du point $B$. Soit $F$ le point d'intersection de $(A C)$ et de la perpendiculaire $(\mathcal{L})$ à $(\Omega B)$ issue de $\Omega:(\Omega B) \cap(\mathcal{L})=\{F\}$ lorsque $(\Omega B)$ et $(A C)$ ne sont pas perpendiculaires. Soit ensuite $S$ le point d'intersection de $(\Omega B)$ avec $(\mathcal{H}):(\Omega B) \cap(\mathcal{H})=\{S\}$ (voir figures 7 et 8 ). Notons $y_{\Omega}$ l'ordonnée de $\Omega$.

Rappelons l'équation de $(A C): y=c x+d R$. La pente de $(\Omega B)$ étant $-y_{\Omega} /(b R)$, l'équation de $(\Omega B)$ est donnée par $y=-\left[y_{\Omega} /(b R)\right] x+y_{\Omega}$ puis celle de $(\mathcal{L})$ est $y=\left[(b R) / y_{\Omega}\right] x+y_{\Omega}$. Les coordonnées $\left(x_{F}, y_{F}\right)$ de $F$ 


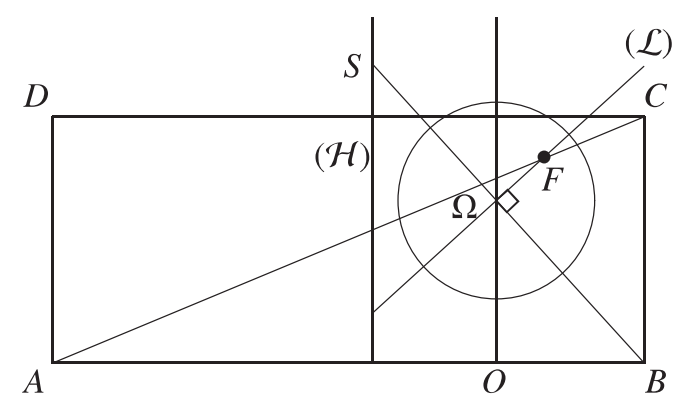

Figure 7. Intersection de $(A C)$ et $(\mathcal{L})$, position haute de $F$.

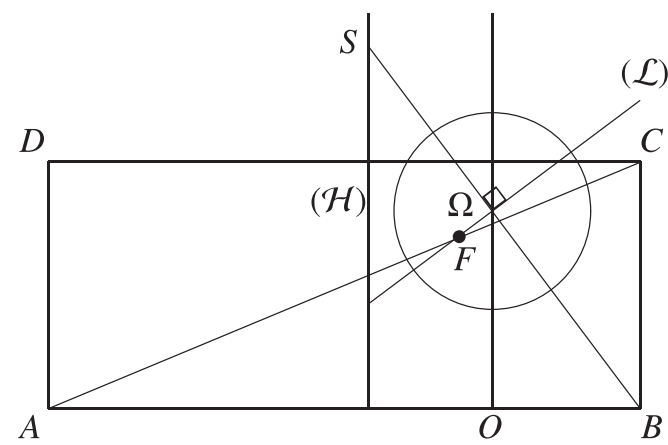

Figure 8. Intersection de $(A C)$ et $(\mathcal{L})$, position basse de $F$.

vérifient les relations $y_{F}=c x_{F}+d R=\left[(b R) / y_{\Omega}\right] x_{F}+y_{\Omega}$ qui donnent

$$
x_{F}=\frac{y_{\Omega}^{2}-d R y_{\Omega}}{c y_{\Omega}-b R}, \quad y_{F}=\frac{c y_{\Omega}^{2}-b d R^{2}}{c y_{\Omega}-b R} .
$$

La condition de triple intersection s'énonce selon $F \in$ $C(\Omega, R)$, soit $x_{F}^{2}+\left(y_{F}-y_{\Omega}\right)^{2}=R^{2}$. Cette équation équivaut, en remplaçant $x_{F}$ et $y_{F}$ par leurs expressions ci-dessus, à

$$
\left(y_{\Omega}^{2}+b^{2} R^{2}\right)\left(y_{\Omega}-d R\right)^{2}=\left(c y_{\Omega}-b R\right)^{2} R^{2} .
$$

On arrive finalement à l'équation de degré 4 d'inconnue $y_{\Omega}$

$$
\begin{aligned}
y_{\Omega}^{4}-2 d R y_{\Omega}^{3}+\left(b^{2}-c^{2}+d^{2}\right) R^{2} y_{\Omega}^{2} & \\
-2(b d-c) b R^{3} y_{\Omega}+\left(d^{2}-1\right) b^{2} R^{4} & =0
\end{aligned}
$$

dont la contre-partie trigonométrique, à rapprocher des équations (1) et (2) en $\cos \theta$ et $\sin \theta$, est obtenue à l'aide de $y_{\Omega}=b R \tan \theta$ :

$$
\begin{aligned}
b^{2} \tan ^{4} \theta- & 2 b d \tan ^{3} \theta+\left(b^{2}-c^{2}+d^{2}\right) \tan ^{2} \theta \\
- & -2(b d-c) \tan \theta+\left(d^{2}-1\right)=0 .
\end{aligned}
$$

On tire ensuite les ordonnées des sommets correspondants, notés $S_{0}^{+}$et $S_{0}^{-}$, à l'aide de la relation d'homothétie $y_{S}=[(a+b) / b] y_{\Omega}$, qui répondra finalement au problème posé (voir figures 9 et 10 ).

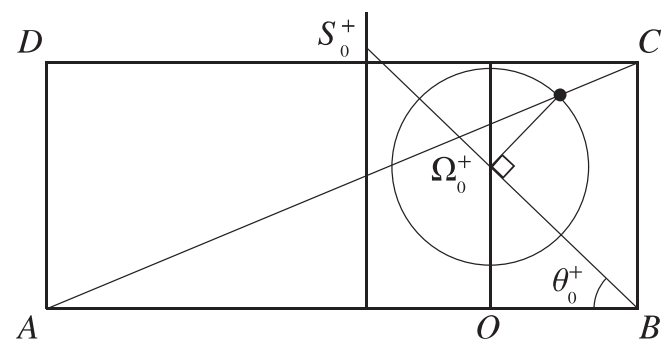

Figure 9. Première solution.

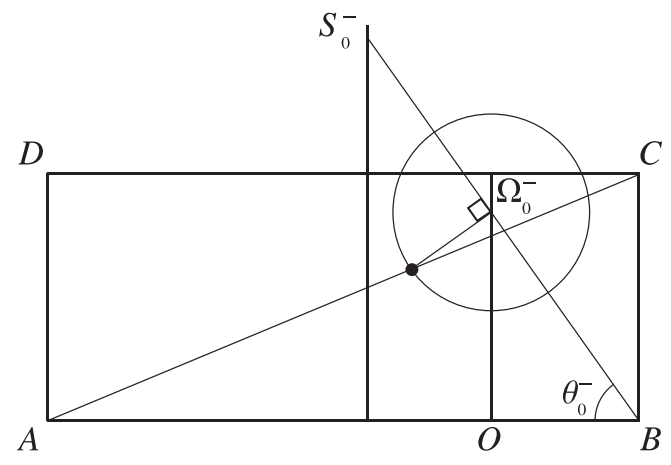

Figure 10. Deuxième solution.

Dans le contexte de la pyramide de Kheops, les deux équations précédentes s'écrivent

$$
4 y_{\Omega}^{4}-12 R y_{\Omega}^{3}+12 R^{2} y_{\Omega}^{2}-8 R^{3} y_{\Omega}+5 R^{4}=0
$$

et

$$
4 \tan ^{4} \theta-12 \tan ^{3} \theta+12 \tan ^{2} \theta-8 \tan \theta+5=0 .
$$

Maple fournit les valeurs numériques

$$
\tan \theta_{0}^{+} \approx 1.277206379 \text { et } \tan \theta_{0}^{-} \approx 1.604572949
$$

puis les angles correspondants (identiques à ceux obtenus dans la section III)

$$
\theta_{0}^{+} \approx 0.906533066 \mathrm{rd} \text { et } \theta_{0}^{-} \approx 1.013478912 \mathrm{rd} \text {. }
$$

\section{IV.2 Deuxième méthode}

D'autres méthodes analytiques équivalentes à la précédente pourraient être envisageables. La suivante requiert plus de calculs que la première méthode, mais nous suggérera une construction géométrique pour situer les sommets recherchés.

Le cercle $C(\Omega, R)$ coulisse le long de l'axe des ordonnées. Soient $E^{+}$et $E^{-}$les points d'intersection de $C(\Omega, R)$ avec $(A C): C(\Omega, R) \cap(A C)=\left\{E^{+}, E^{-}\right\}$, ce qui suppose que le cercle se déplace entre deux positions tangentes à la diagonale $(A C)$, positions extrêmes pour lesquelles $E^{+}=E^{-}$. Les segments 


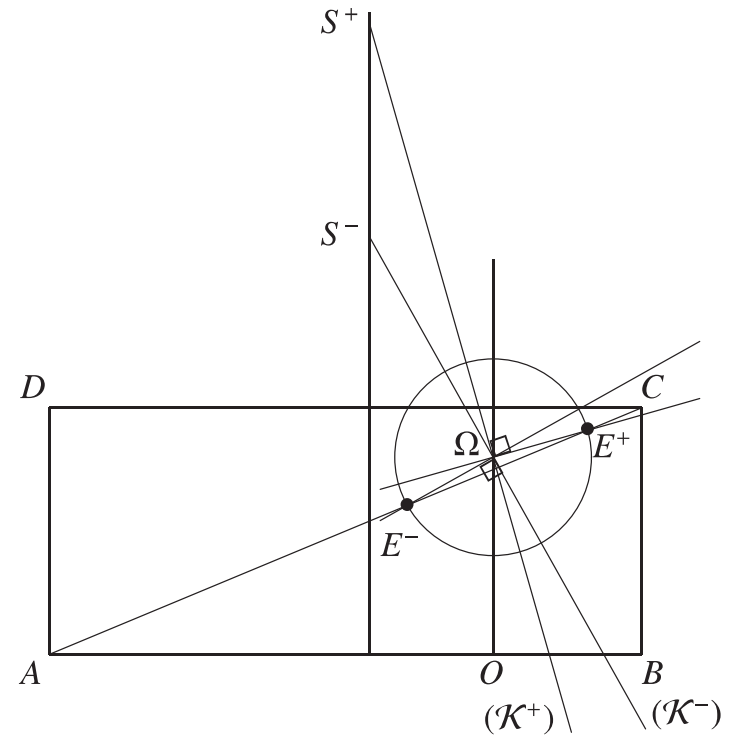

Figure 11. Intersection du cercle et de la diagonale.

$\left[\Omega, E^{+}\right]$et $\left[\Omega, E^{-}\right]$coulissent le long des deux glissières portées par l'axe des ordonnées $(O y)$ et la diagonale $(A C)$ à l'instar d'une porte de garage (à glissières qui ne seraient pas d'équerre). On trace les perpendiculaires $\left(\mathcal{K}^{+}\right)$et $\left(\mathcal{K}^{-}\right)$issues de $\Omega$ à $\left(\Omega E^{+}\right)$ et $\left(\Omega E^{-}\right)$respectivement, puis on note $S^{+}$et $S^{-}$les points d'intersection respectifs de $(\mathcal{H})$ avec $\left(\mathcal{K}^{+}\right)$et $\left(\mathcal{K}^{-}\right):(\mathcal{H}) \cap\left(\mathcal{K}^{+}\right)=\left\{S^{+}\right\}$et $(\mathcal{H}) \cap\left(\mathcal{K}^{-}\right)=\left\{S^{-}\right\}($voir figure 11).

On cherche les centres $\Omega_{0}^{+}$et $\Omega_{0}^{-}$pour que $\left(\mathcal{K}^{+}\right)$et $\left(\mathcal{K}^{-}\right)$passent par $B$ (pas simultanément). Les sommets recherchés seront notés $S_{0}^{+}$et $S_{0}^{-}$.

Les équations de $(A C)$ et $C(\Omega, R)$ étant respectivement $y=c x+d R$ et $x^{2}+\left(y-y_{\Omega}\right)^{2}=R^{2}$, la recherche des points d'intersection $E^{+}$et $E^{-}$revient à résoudre

$\left(c^{2}+1\right) x^{2}-2 c\left(y_{\Omega}-d R\right) x+\left[y_{\Omega}^{2}-2 d R y_{\Omega}+\left(d^{2}-1\right) R^{2}\right]=0$.

Le discriminant de cette équation est

$$
\begin{aligned}
\Delta & =\left(c^{2}-d^{2}+1\right) R^{2}+2 d R y_{\Omega}-y_{\Omega}^{2} \\
& =\left(c^{2}+1\right) R^{2}-\left(y_{\Omega}-d R\right)^{2} .
\end{aligned}
$$

Il est positif ou nul pour les valeurs de $y_{\Omega}$ comprises entre $\left(d-\sqrt{c^{2}+1}\right) R$ et $\left(d+\sqrt{c^{2}+1}\right) R$, ces deux valeurs étant associées aux positions extrêmes du cercle tangentes à $(A C)$. Les solutions sont

$$
\begin{aligned}
& x_{E^{+}}=\frac{c\left(y_{\Omega}-d R\right)+\sqrt{\Delta}}{c^{2}+1}, \\
& x_{E^{-}}=\frac{c\left(y_{\Omega}-d R\right)-\sqrt{\Delta}}{c^{2}+1}
\end{aligned}
$$

et les $y$ correspondants $(y=c x+d R)$ sont donnés par

$$
\begin{aligned}
& y_{E^{+}}=\frac{c^{2} y_{\Omega}+d R+c \sqrt{\Delta}}{c^{2}+1}, \\
& y_{E^{-}}=\frac{c^{2} y_{\Omega}+d R-c \sqrt{\Delta}}{c^{2}+1} .
\end{aligned}
$$

Le vecteur $\overrightarrow{\Omega E}$ (on notera $E$ pour $E^{+}$ou $E^{-}$indifféremment) ayant pour coordonnées $\left(x_{E}, y_{E}-y_{\Omega}\right)$, la perpendiculaire $(\mathcal{K})$ (on notera également $(\mathcal{K})$ pour $\left(\mathcal{K}^{+}\right)$ ou $\left.\left(\mathcal{K}^{-}\right)\right)$à $(\Omega E)$ passant par $\Omega$ a pour équation

$$
x_{E} x+\left(y_{E}-y_{\Omega}\right)\left(y-y_{\Omega}\right)=0 .
$$

Le point d'intersection $S$ de $(\mathcal{K})$ avec l'axe $(\mathcal{H})$ d'équation $x=-a R$ a alors pour ordonnée $y_{S}=$ $y_{\Omega}+\left(x_{E} a R\right) /\left(y_{E}-y_{\Omega}\right)$. Les ordonnées des sommets $S^{+}$et $S^{-}$associés aux points $E^{+}$et $E^{-}$ont ainsi pour expressions, au vu de (5), (6), (7) et (8) :

$$
\begin{aligned}
& y_{S^{+}}=\frac{y_{\Omega}^{2}-(a c+d) R y_{\Omega}+a c d R^{2}-\left(c y_{\Omega}+a R\right) \sqrt{\Delta}}{y_{\Omega}-d R-c \sqrt{\Delta}}, \\
& y_{S^{-}}=\frac{y_{\Omega}^{2}-(a c+d) R y_{\Omega}+a c d R^{2}+\left(c y_{\Omega}+a R\right) \sqrt{\Delta}}{y_{\Omega}-d R+c \sqrt{\Delta}} .
\end{aligned}
$$

Les sommets $S$ recherchés de la pyramide sont les points pour lesquels la droite $(\mathcal{K})$ passe par le point $B$ de la base rectangulaire. Reportant les coordonnées $(b R, 0)$ de $B$ ainsi que la relation $y_{E}=c x_{E}+d R$ dans l'équation (9) de $(\mathcal{K})$, on est amené à résoudre l'équation $\left(c y_{\Omega}-b R\right) x_{E}=y_{\Omega}^{2}-d R y_{\Omega}$ d'inconnue $y_{\Omega}$. En utilisant les expressions (5) et (6) de $x_{E^{ \pm}}$, cela donne les équivalences ci-dessous :

$$
\begin{gathered}
\left(c y_{\Omega}-b R\right)\left[c\left(y_{\Omega}-d R\right) \pm \sqrt{\Delta}\right] \\
=\left(c^{2}+1\right)\left(y_{\Omega}^{2}-d R y_{\Omega}\right) \\
\Longleftrightarrow\left(c^{2}+1\right)\left(y_{\Omega}^{2}-d R y_{\Omega}\right)-c\left(c y_{\Omega}-b R\right)\left(y_{\Omega}-d R\right) \\
= \pm\left(c y_{\Omega}-b R\right) \sqrt{\Delta}
\end{gathered}
$$

$$
\begin{aligned}
\Longleftrightarrow & \left(y_{\Omega}^{2}+(b c-d) R y_{\Omega}-b c d R^{2}\right)^{2} \\
& =\left(c y_{\Omega}-b R\right)^{2}\left(-y_{\Omega}^{2}+2 d R y_{\Omega}+\left(c^{2}-d^{2}+1\right) R^{2}\right) \\
\Longleftrightarrow & y_{\Omega}^{4}-2 d R y_{\Omega}^{3}+\left(b^{2}-c^{2}+d^{2}\right) R^{2} y_{\Omega}^{2} \\
& \quad-2(b d-c) b R^{3} y_{\Omega}+\left(d^{2}-1\right) b^{2} R^{4}=0 .
\end{aligned}
$$

On retrouve ainsi l'équation (3) obtenue dans la soussection IV.1.

\section{Approche algébrique}

Dans cette section, nous nous intéressons au cas particulier de la pyramide de Kheops et prouvons que les deux sommets $S_{0}^{+}$et $S_{0}^{-}$ne sont pas constructibles 
à la règle et au compas. Pour cela, nous invoquons une propriété que l'on peut trouver dans le livre de J.C. Carréga [1] (exercice 24) et qui s'énonce comme suit :

Si un polynôme de degré 4, sans terme de degré 3, à coefficients rationnels, ne peut être décomposé en un produit de polynômes non constants à coefficients rationnels et si son polynôme résolvant ne peut non plus être ainsi décomposé, alors les racines du polynôme initial ne sont pas constructibles à la règle et au compas.

Nous considérons ici l'équation (4) réécrite, en posant $\tan \theta=x$, selon

$$
4 x^{4}-12 x^{3}+12 x^{2}-8 x+5=0 .
$$

Dans la sous-section V.1, nous construisons l'équation résolvante associée à (10) à partir de laquelle, couplée avec (11), nous déduirons la propriété de non-constructibilité à la règle et au compas. Dans la sous-section V.2, nous présentons, par souci de complétude, la résolution totale de l'équation (11).

\section{V.1 Équation résolvante}

En effectuant la translation adéquate $x=y+3 / 4$, on transforme (10) en l'équation sans terme de degré 3

$$
256 y^{4}-96 y^{2}-224 y+125=0 .
$$

Introduisons les fonctions symétriques élémentaires des racines $y_{1}, y_{2}, y_{3}, y_{4}$ de (11):

$$
\begin{aligned}
& \sigma_{1}=y_{1}+y_{2}+y_{3}+y_{4}, \\
& \sigma_{2}=y_{1} y_{2}+y_{1} y_{3}+y_{1} y_{4}+y_{2} y_{3}+y_{2} y_{4}+y_{3} y_{4}, \\
& \sigma_{3}=y_{1} y_{2} y_{3}+y_{1} y_{2} y_{4}+y_{1} y_{3} y_{4}+y_{2} y_{3} y_{4}, \\
& \sigma_{4}=y_{1} y_{2} y_{3} y_{4} .
\end{aligned}
$$

Ici $\sigma_{1}=0, \sigma_{2}=-96 / 256=-3 / 8, \sigma_{3}=224 / 256=$ $7 / 8, \sigma_{4}=125 / 256$. Introduisons également les quantités (classiques dans la théorie de l'équation du quatrième degré), en tenant compte de la relation $\sigma_{1}=0$, suivantes :

$$
\begin{aligned}
& z_{1}=-\left(y_{1}+y_{2}\right)\left(y_{3}+y_{4}\right)=\left(y_{1}+y_{2}\right)^{2}, \\
& z_{2}=-\left(y_{1}+y_{3}\right)\left(y_{2}+y_{4}\right)=\left(y_{1}+y_{3}\right)^{2}, \\
& z_{3}=-\left(y_{1}+y_{4}\right)\left(y_{2}+y_{3}\right)=\left(y_{1}+y_{4}\right)^{2} .
\end{aligned}
$$

On a clairement

$$
z_{1}+z_{2}+z_{3}=-2 \sigma_{2}=\frac{3}{4} .
$$

Ensuite, en écrivant

$\left(y_{1}+y_{2}\right)\left(y_{1}+y_{3}\right)=y_{1}\left(y_{1}+y_{2}+y_{3}\right)+y_{2} y_{3}=y_{2} y_{3}-y_{1} y_{4}$ ainsi que, de manière analogue, $\left(y_{1}+y_{2}\right)\left(y_{1}+y_{4}\right)=$ $y_{2} y_{4}-y_{1} y_{3}$ et $\left(y_{1}+y_{3}\right)\left(y_{1}+y_{4}\right)=y_{3} y_{4}-y_{1} y_{2}$, on obtient

$$
\begin{aligned}
& z_{1} z_{2}=y_{1}^{2} y_{4}^{2}+y_{2}^{2} y_{3}^{2}-2 \sigma_{4}, \\
& z_{1} z_{3}=y_{1}^{2} y_{3}^{2}+y_{2}^{2} y_{4}^{2}-2 \sigma_{4}, \\
& z_{2} z_{3}=y_{1}^{2} y_{2}^{2}+y_{3}^{2} y_{4}^{2}-2 \sigma_{4},
\end{aligned}
$$

puis

$$
z_{1} z_{2}+z_{1} z_{3}+z_{2} z_{3}=\sigma_{2}^{2}-4 \sigma_{4}=-\frac{29}{16} .
$$

Enfin, observant que

$$
\begin{aligned}
\left(y_{1}+y_{2}\right) & \left(y_{1}+y_{3}\right)\left(y_{1}+y_{4}\right) \\
= & y_{1}^{2}\left(y_{1}+y_{2}+y_{3}+y_{4}\right) \\
& +y_{1} y_{2} y_{3}+y_{1} y_{2} y_{4}+y_{1} y_{3} y_{4}+y_{2} y_{3} y_{4} \\
= & \sigma_{3},
\end{aligned}
$$

on trouve immédiatement

$$
z_{1} z_{2} z_{3}=\sigma_{3}^{2}=\frac{49}{64} .
$$

Par conséquent, les nombres $z_{1}, z_{2}, z_{3}$ sont les solutions de l'équation résolvante (de Descartes) associée à (11)

$$
64 z^{3}-48 z^{2}-116 z-49=0 .
$$

Cherchons à présent si (13) admet une racine de la forme $r / s$ avec $r, s$ deux entiers premiers entre eux et $s>0$ :

$$
64 r^{3}-48 r^{2} s-116 r s^{2}-49 s^{3}=0 .
$$

On voit que $r$ doit diviser $49=7^{2}$ et que $s$ doit diviser $64=2^{6}$, donc $r \in\{ \pm 1, \pm 7, \pm 49\}$ et $s \in$ $\{1,2,4,8,16,32,64\}$. Il est facile de vérifier qu'aucune des 42 fractions ainsi obtenues n'est solution de (13), donc le polynôme contenu dans (13) ne peut pas se décomposer en le produit d'un polynôme de degré 1 et un de degré 2 à coefficients rationnels.

Cherchons ensuite si (11) admet une racine de la forme $p / q$ avec $p, q$ deux entiers premiers entre eux et $q>0$ :

$$
256 p^{4}-96 p^{2} q^{2}-224 p q^{3}+125 q^{4}=0 .
$$

L'entier $p$ doit donc diviser $125=5^{3}$ et $q$ doit diviser $256=2^{8}$, donc $p \in\{ \pm 1, \pm 5, \pm 25, \pm 125\}$ et $q \in\{1,2,4,8,16,32,64,128,256\}$. Aucune des 72 fractions candidates ne convient, donc le polynôme contenu dans (11) ne peut pas se décomposer en le produit d'un polynôme de degré 1 et un de degré 3 à coefficients rationnels. 
Il reste à voir s'il pourrait se décomposer en le produit de deux polynômes de degré 2 à coefficients rationnels. Une telle décompostion s'écrirait

$$
\begin{aligned}
256 y^{4}-96 y^{2}-224 y+125 & \\
& =256\left(y^{2}-\alpha_{1} y+\beta_{1}\right)\left(y^{2}-\alpha_{2} y+\beta_{2}\right),
\end{aligned}
$$

pour des rationnels $\alpha_{1}, \beta_{1}, \alpha_{2}, \beta_{2}$. En identifiant, on trouve $\alpha_{2}=-\alpha_{1}, \alpha_{1} \alpha_{2}+\beta_{1}+\beta_{2}=-96 / 256=-3 / 8$, $\alpha_{1} \beta_{2}+\alpha_{2} \beta_{1}=224 / 256=7 / 8$ et $\beta_{1} \beta_{2}=125 / 256$. De ces relations, on tire $\alpha_{1}=-\alpha_{2}=\alpha$ disons, puis $\beta_{1}+\beta_{2}=\alpha^{2}-3 / 8$ et $\beta_{1}-\beta_{2}=-7 /(8 \alpha)$. On en déduit que

$$
\beta_{1}=\frac{1}{2}\left(\alpha^{2}-\frac{3}{8}-\frac{7}{8 \alpha}\right)=\frac{8 \alpha^{3}-3 \alpha-7}{16 \alpha}
$$

et

$$
\beta_{2}=\frac{1}{2}\left(\alpha^{2}-\frac{3}{8}+\frac{7}{8 \alpha}\right)=\frac{8 \alpha^{3}-3 \alpha+7}{16 \alpha} .
$$

En reportant ces expressions dans la relation $\beta_{1} \beta_{2}=$ $125 / 256$, on obtient l'équation de degré 3 pour $\alpha^{2}$ suivante :

$$
64 \alpha^{6}-48 \alpha^{4}-116 \alpha^{2}-49=0 .
$$

Remarquons que l'équation (14) n'est autre que la version tricarrée de l'équation résolvante (13) (qui n'a pas de solution rationnelle) et donc le polynôme contenu dans (11) ne peut pas se décomposer en le produit de deux polynômes de degré 2 à coefficients rationnels.

La propriété de non constructibilité à la règle et au compas est finalement prouvée.

\section{V.2 Résolution complète}

La translation adéquate $z=w+1 / 4$ transforme (13) en l'équation de degré 3 sans terme de degré 2

$$
w^{3}-2 w-5 / 4=0 .
$$

La méthode de Cardan fournit les solutions

$$
\begin{aligned}
& w_{1}=u+v, \\
& w_{2}=u j+v \bar{j}=-\frac{1}{2}[(u+v)-i \sqrt{3}(u-v)], \\
& w_{3}=u \bar{j}+v j=-\frac{1}{2}[(u+v)+i \sqrt{3}(u-v)],
\end{aligned}
$$

où $u$ et $v$ vérifient le système $u^{3}+v^{3}=5 / 4$ et $u v=2 / 3$, ce qui donne

$$
\begin{aligned}
& u=\frac{1}{6} \sqrt[3]{135+3 \sqrt{489}} \approx 0.9768445587 \\
& v=\frac{1}{6} \sqrt[3]{135-3 \sqrt{489}} \approx 0.6824695506
\end{aligned}
$$

Rappelons que $z_{1}=\left(y_{1}+y_{2}\right)^{2}, z_{2}=\left(y_{1}+y_{3}\right)^{2}$, $z_{3}=\left(y_{2}+y_{3}\right)^{2}$, ici avec $z_{k}=w_{k}+1 / 4,1 \leqslant k \leqslant 3$. Les $y_{1}, y_{2}, y_{3}, y_{4}$ sont donc solutions du système $y_{1}+y_{2}=$ $\zeta_{1}, y_{1}+y_{3}=\zeta_{2}, y_{2}+y_{3}=\zeta_{3}$ où $\zeta_{1}, \zeta_{2}, \zeta_{3}$ sont des racines carrées de $z_{1}, z_{2}, z_{3}$ respectivement, le choix des signes de celles-ci devant satisfaire une contrainte qui va être précisée ci-dessous. En effet, se référant à (12), on trouve que

$$
\begin{aligned}
& \left(y_{1}+y_{2}\right)\left(y_{1}+y_{3}\right)\left(y_{2}+y_{3}\right) \\
& =-\left(y_{1}+y_{2}\right)\left(y_{1}+y_{3}\right)\left(y_{1}+y_{4}\right)=-7 / 8
\end{aligned}
$$

et la condition sur les signes de $\zeta_{1}, \zeta_{2}, \zeta_{3}$ est $\zeta_{1} \zeta_{2} \zeta_{3}=$ $-7 / 8$. Rappelons maintenant que les racines carrées d'un nombre complexe $\xi+i \eta$ sont

$$
\pm\left[\sqrt{\frac{1}{2}\left(\sqrt{\xi^{2}+\eta^{2}}+\xi\right)}+i \operatorname{sgn}(\eta) \sqrt{\frac{1}{2}\left(\sqrt{\xi^{2}+\eta^{2}}-\xi\right)}\right] .
$$

Posons alors $\rho=\left|z_{2}\right|=\left|z_{3}\right|$. On a, puisque $u v=2 / 3$,

$$
\begin{aligned}
\rho^{2} & =\left(\frac{u+v}{2}-\frac{1}{4}\right)^{2}+\left(\sqrt{3} \frac{u-v}{2}\right)^{2} \\
& =u^{2}+v^{2}-\frac{u+v}{4}-\frac{29}{48} \approx 0.4009947843 .
\end{aligned}
$$

On choisira par exemple (on vérifie que $\zeta_{1} \zeta_{2} \zeta_{3}<0$ )

$$
\begin{aligned}
& \zeta_{1}=-\sqrt{u+v+1 / 4} \\
& \zeta_{2}=\frac{1}{2}[\sqrt{2 \rho-(u+v-1 / 2)}+i \sqrt{2 \rho+(u+v-1 / 2)}], \\
& \zeta_{3}=\frac{1}{2}[\sqrt{2 \rho-(u+v-1 / 2)}-i \sqrt{2 \rho+(u+v-1 / 2)}] .
\end{aligned}
$$

On a alors les égalités $\zeta_{2}+\zeta_{3}=\sqrt{2 \rho-(u+v-1 / 2)}$ et $\zeta_{2}-\zeta_{3}=i \sqrt{2 \rho+(u+v-1 / 2)}$, et enfin les solutions de (11) s'écrivent (on fera après $x_{k}=y_{k}+3 / 4,1 \leqslant$ $k \leqslant 4$, pour récupérer les solutions de (10))

$$
\begin{aligned}
y_{1} & =\frac{1}{2}\left(\zeta_{1}+\zeta_{2}-\zeta_{3}\right) \\
& =\frac{1}{2}[-\sqrt{u+v+1 / 4}+i \sqrt{2 \rho+(u+v-1 / 2)}], \\
y_{2} & =\frac{1}{2}\left(\zeta_{1}-\zeta_{2}+\zeta_{3}\right) \\
& =\frac{1}{2}[-\sqrt{u+v+1 / 4}-i \sqrt{2 \rho+(u+v-1 / 2)}], \\
y_{3} & =\frac{1}{2}\left(-\zeta_{1}+\zeta_{2}+\zeta_{3}\right) \\
& =\frac{1}{2}[\sqrt{u+v+1 / 4}+\sqrt{2 \rho-(u+v-1 / 2)}], \\
y_{4} & =\frac{1}{2}\left(-\zeta_{1}-\zeta_{2}-\zeta_{3}\right) \\
& =\frac{1}{2}[\sqrt{u+v+1 / 4}-\sqrt{2 \rho-(u+v-1 / 2)}] .
\end{aligned}
$$


Maple donne les valeurs numériques de $\zeta_{1}, \zeta_{2}, \zeta_{3}$ :

$$
\begin{aligned}
& \zeta_{1} \approx 1.381779327, \\
& \zeta_{2} \approx 0.1636832850+0.7787485298 i, \\
& \zeta_{3} \approx 0.1636832850-0.7787485298 i,
\end{aligned}
$$

puis celles des solutions de (10) :

$$
\begin{aligned}
& x_{1} \approx 0.0591103365+0.7787485298 i, \\
& x_{2} \approx 0.0591103365-0.7787485298 i, \\
& x_{3} \approx 1.604572948, \\
& x_{4} \approx 1.277206378 .
\end{aligned}
$$

\section{Une construction géométrique}

Malgré l'impossibilité de tracer les sommets $S_{0}^{+}$ et $S_{0}^{-}$à la règle et au compas, nous proposons une construction originale permettant d'obtenir empiriquement ces points comme intersection d'une certaine courbe que nous précisons ci-dessous avec une droite bien spécifique.

En reprenant les notations de la section III, on écrit la tangente des angles $\theta_{0}^{ \pm}$associés aux sommets $S_{0}^{ \pm}$de deux façons :

$$
\tan \theta_{0}^{ \pm}=\frac{y_{S_{0}^{ \pm}}}{(a+b) R}=\frac{y_{\Omega_{0}^{ \pm}}}{b R}
$$

qui fournit l'égalité des rapports (c'est aussi le théorème de Thalès!) $y_{S_{0}^{ \pm}} / y_{\Omega_{0}^{ \pm}}=(a+b) / b$, soit encore

$$
y_{S_{0}^{ \pm}}=\left(1+\frac{a}{b}\right) y_{\Omega_{0}^{ \pm}} \text {. }
$$

En utilisant les résultats de la sous-section IV.2 (voir les ordonnées de $y_{S^{ \pm}}$), cette observation se formule selon $f^{ \pm}(y)=g(y)$ où l'on a posé $f^{ \pm}(y)=f_{1}^{ \pm}(y) / f_{2}^{ \pm}(y)$ avec

$$
\begin{aligned}
f_{1}^{ \pm}(y)= & y^{2}-(a c+d) R y+a c d R^{2} \\
& \mp(c y+a R) \sqrt{\left(c^{2}-d^{2}+1\right) R^{2}+2 d R y-y^{2}} \\
f_{2}^{ \pm}(y)= & y-d R \mp c \sqrt{\left(c^{2}-d^{2}+1\right) R^{2}+2 d R y-y^{2}}
\end{aligned}
$$

et

$$
g(y)=\left(1+\frac{a}{b}\right) y .
$$

Notre construction repose précisément sur les équations $f^{+}(y)=g(y)$ et $f^{-}(y)=g(y)$, lesquelles reviennent à chercher graphiquement les points d'intersection des courbes représentatives de $f^{+}$et $f^{-}$avec la droite associée à $g$. Notre méthode de construction consiste alors à tracer les courbes $f^{+}, f^{-}$et $g$ selon le procédé décrit ci-dessous.

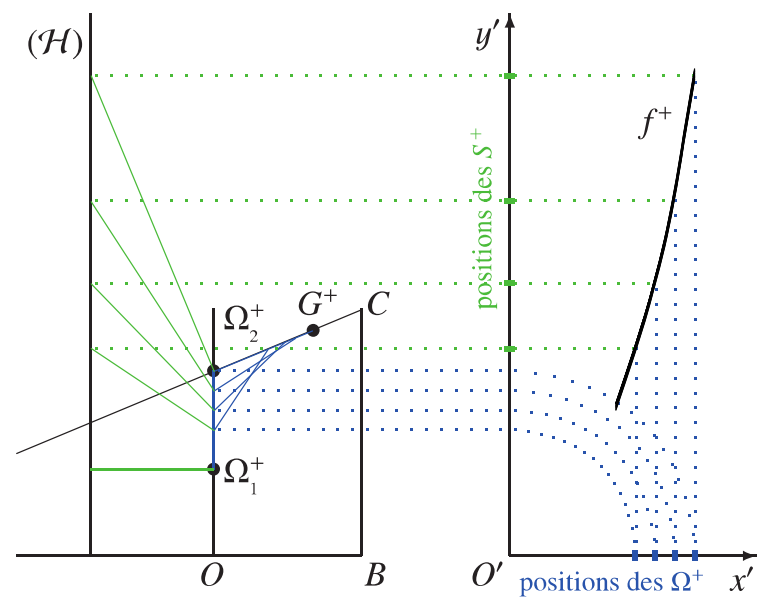

Figure 12. Courbe représentative de $f^{+}$.

- Nous référant à la méthode exposée dans la sous-section IV.2, nous traçons une succession de segments de longueur $R$ dont les extrémités inférieures coulissent le long d'un segment vertical $\left[\Omega_{1}^{+}, \Omega_{2}^{+}\right]$et les extrémités supérieures le long d'un segment oblique $\left[\Omega_{2}^{+}, G^{+}\right]$. Le point $\Omega_{2}^{+}$est l'intersection de l'axe $(O y)$ et de la diagonale $(A C)$, et les points $\Omega_{1}^{+}$et $G^{+}$ se trouvent à la distance $R$ de $\Omega_{2}^{+}, \Omega_{1}^{+}$étant situé sur $(O y)$ au-dessous de $\Omega_{2}^{+}$et $G^{+}$sur $(A C)$ à droite de $\Omega_{2}^{+}$(voir figure 12). Les extrémités inférieures sont les centres successifs $\Omega$ des cercles coulissants et nous choisissons leurs ordonnées $y_{\Omega}$ régulièrement espacées. Nous rabattons alors en abscisses à l'aide d'une rotation d'angle droit $(-\pi / 2)$ sur un graphique parallèle de repère $\left(O^{\prime} x^{\prime} y^{\prime}\right)$ les ordonnées successives $y_{\Omega}$. Les ordonnées $y_{S^{+}}$des sommets $S^{+}$ associés aux $\Omega$ selon la même méthode du IV.2 (rappelons que les $S^{+}$sont les intersections de la hauteur $(\mathcal{H})$ avec les perpendiculaires issues de $\Omega$ aux segments coulissants) sont reportées en ordonnées sur ce nouveau graphique. Ce procédé fournit un échantillon de la courbe représentative de $f^{+}$(voir figure 12), appelée polyligne en CAO. Nous construisons de manière similaire la courbe représentative de $f^{-}$à partir d'une succession de segments coulissant entre un segment vertical $\left[\Omega_{1}^{-}, \Omega_{2}^{-}\right]$et un segment oblique $\left[\Omega_{1}^{-}, G^{-}\right]$. Dans ce dernier cas, $\Omega_{1}^{-}$ est l'intersection de $(O y)$ et $(A C)$, puis $\Omega_{2}^{-}$et $G^{-}$ se trouvent à la distance $R$ de $\Omega_{1}^{-}, \Omega_{2}^{-}$étant sur $(O y)$ au-dessus de $\Omega_{1}^{-}$et $G^{-}$sur $(A C)$ à gauche de $\Omega_{1}^{-}$(voir figure 13).

- Ensuite, nous construisons la droite associée à $g$ en rabattant la distance horizontale $(a+b) R$ (distance entre le point $B$ et la hauteur $(\mathcal{H})$ ) en 

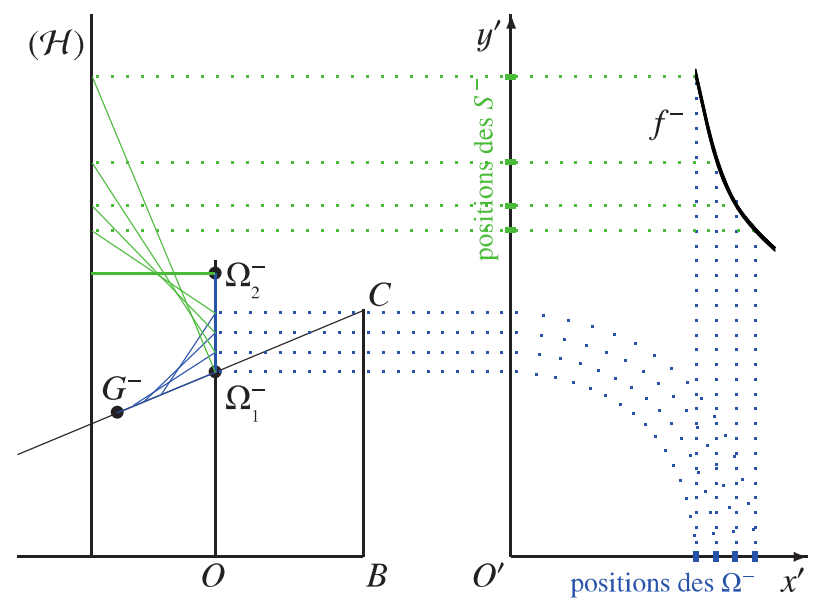

Figure 13. Courbe représentative de $f^{-}$.
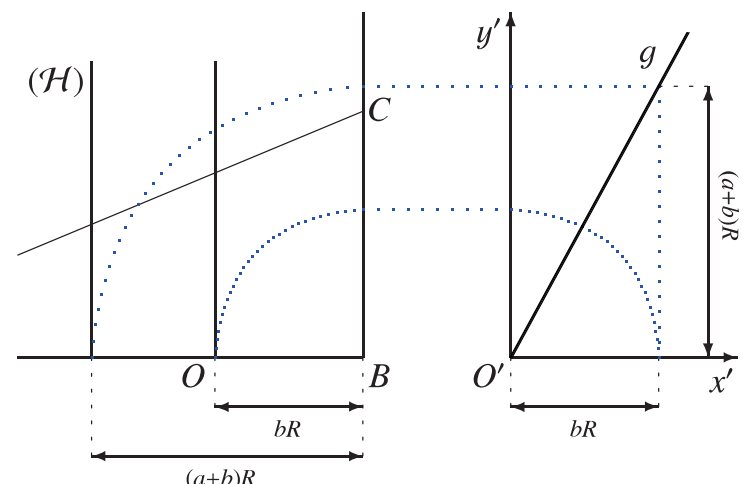

Figure 14. Construction de la droite de pente $1+a / b$.
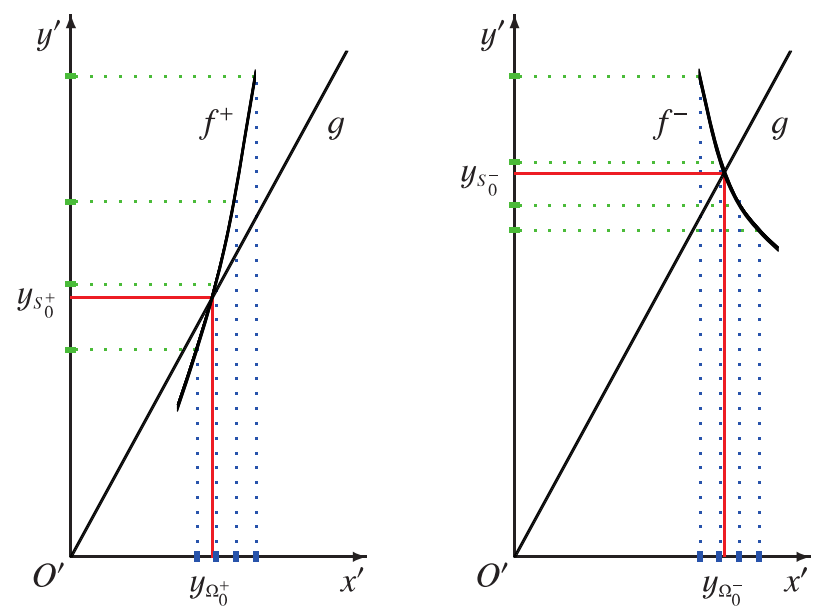

Figure 15. Construction des sommets $S_{0}^{+}$et $S_{0}^{-}$.

une distance verticale par une rotation d'angle droit $(-\pi / 2)$ sur le même graphique parallèle $\left(O^{\prime} x^{\prime} y^{\prime}\right)$, ainsi que la distance horizontale $b R$ (distance entre $O$ et $B$ ) par une rotation d'angle plat $(-\pi)$ pour cette dernière (voir figure 14$)$.

- Enfin, les intersections des courbes représentatives de $f^{+}$et $f^{-}$avec la droite d'équation $g$ fournissent les sommets $S_{0}^{+}$et $S_{0}^{-}$(voir figure 15).
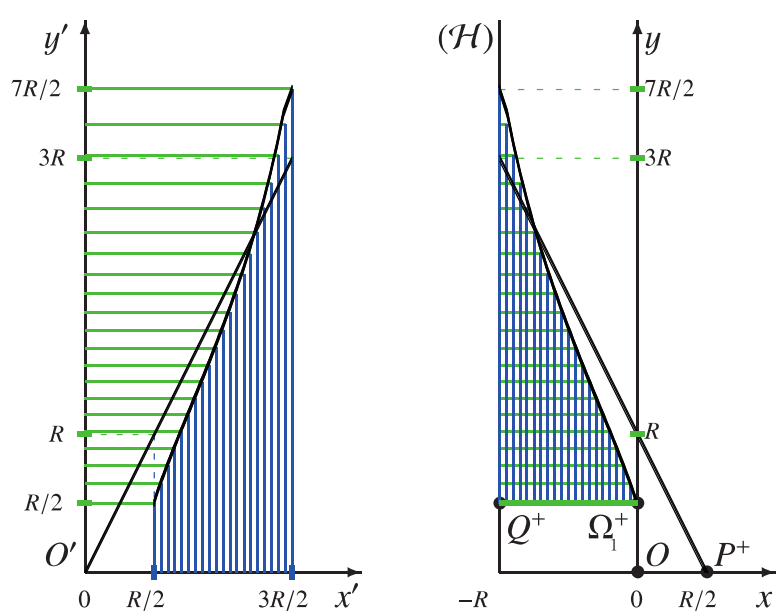

Figure 16. Cas de la pyramide de Kheops : symétrie.

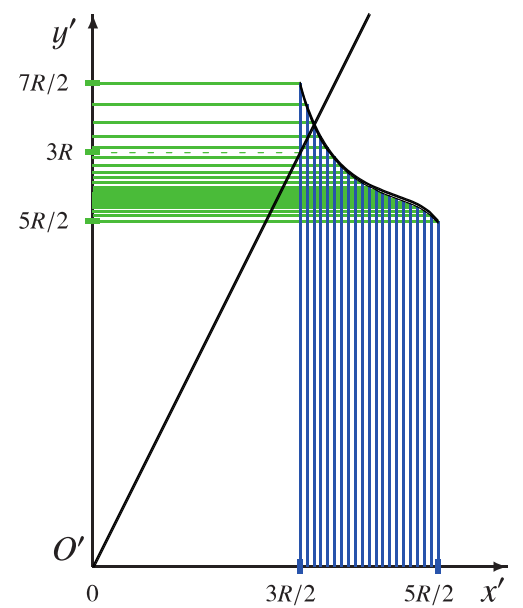

Figure 17. Cas de la pyramide de Kheops.

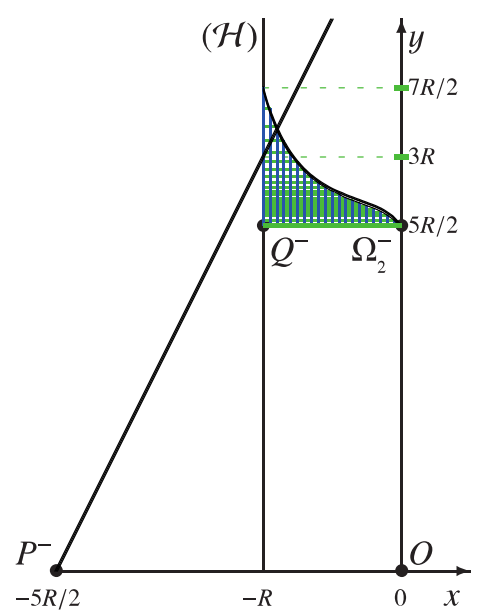

Figure 18. Cas de la pyramide de Kheops : translation.

Nous avons jugé intéressant et esthétique d'inclure la construction ainsi décrite au graphique initial, comme cela nous a été suggéré par A. Dufour. Cela donne les graphiques des figures 19 et 20 illustrant le cas de la pyramide de Kheops. Nous détaillons davantage la construction de $S_{0}^{+}$et $S_{0}^{-}$dans ce dernier cas. 


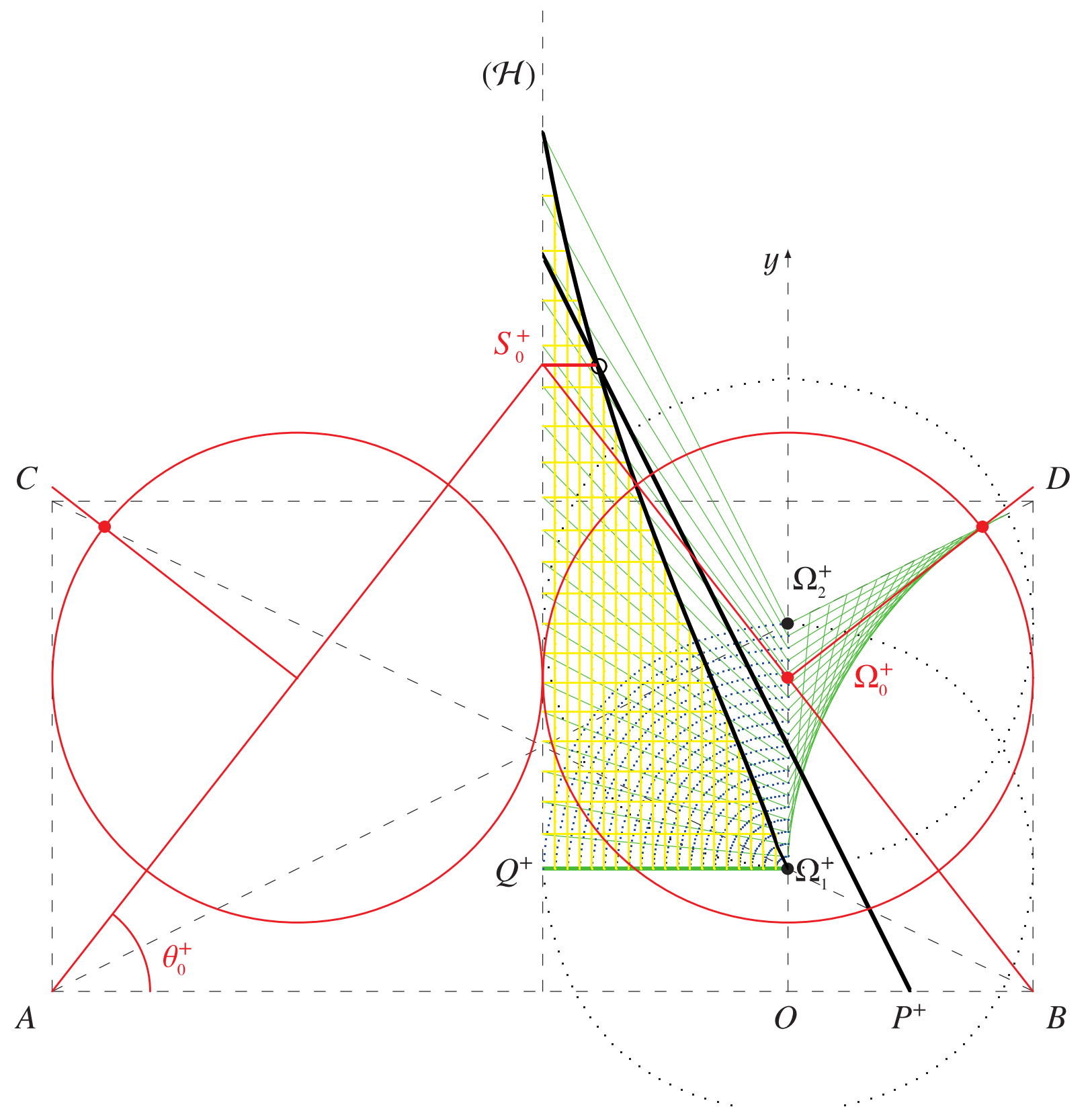

Figure 19. La pyramide de Kheops, première construction.

Les fonctions $f_{1}^{ \pm}$et $f_{2}^{ \pm}$prennent alors la forme particulière

$$
\begin{aligned}
2 f_{1}^{ \pm}(y)= & 2 y^{2}-4 R y+\frac{3}{2} R^{2} \\
& \mp(y+2 R) \sqrt{-y^{2}+3 R y-R^{2}}, \\
2 f_{2}^{ \pm}(y)= & 2 y-3 R \mp \sqrt{-y^{2}+3 R y-R^{2}} .
\end{aligned}
$$

- Première construction. Le cercle mobile coulisse entre deux positions correspondant aux centres $\Omega_{1}^{+}$d'ordonnée $R / 2$ et $\Omega_{2}^{+}$d'ordonnée $3 R / 2$. Pour cette situation, il nous semble préférable de rabattre les ordonnées $y_{\Omega}$ (nous avons choisi une vingtaine de graduations équidistantes) sur le segment, noté $\left[Q^{+}, \Omega_{1}^{+}\right]$, d'équation $y=R / 2$ compris entre la hauteur $(\mathcal{H})$ et l'axe $(O y)$ par une rotation de centre $\Omega_{1}^{+}$et d'angle $+\pi / 2$ (voir figure 16 ), angle opposé à celui de la construction générale décrite ci-dessus (voir figure 12). Le pied de la droite oblique se trouvant en $O^{\prime}$ sur la figure intermédiaire 16 , dessin de gauche, à la distance $R / 2$ à gauche de la première graduation se retrouve en $P_{1}$ à la distance $R / 2$ à droite de $O$ sur la figure initiale 16, dessin de droite. De plus, la droite oblique et la courbe d'intérêt se retrouvent inversées sur ce dernier dessin. Nous obtenons ainsi le graphique de la figure 19. 


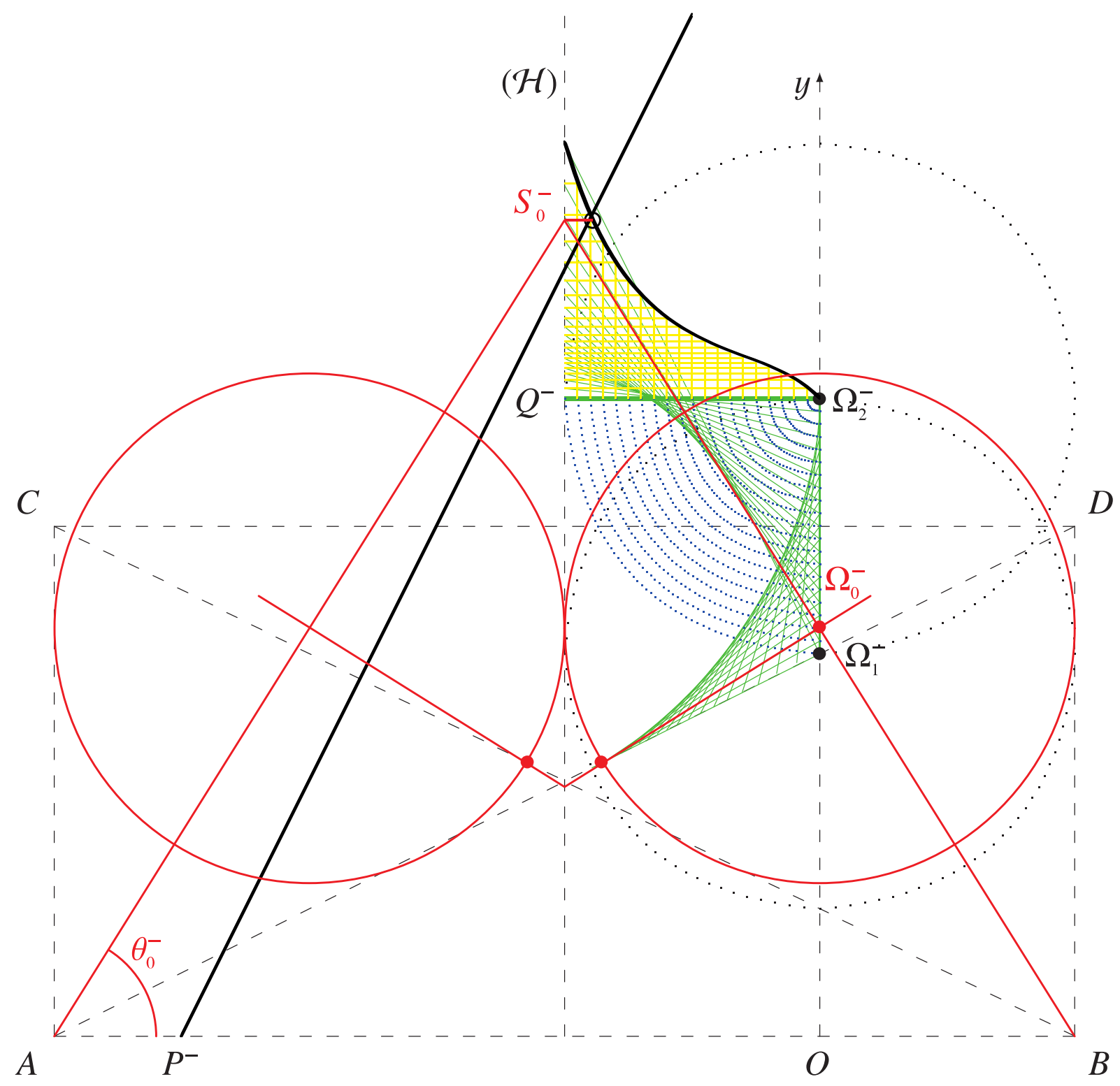

Figure 20. La pyramide de Kheops, deuxième construction.

- Deuxième construction. Le cercle mobile coulisse entre deux positions correspondant aux centres $\Omega_{1}^{-}$d'ordonnée $3 R / 2$ et $\Omega_{2}^{-}$d'ordonnée $5 R / 2$. Dans ce cas, nous rabattons les ordonnées $y_{\Omega}$ sur le segment d'équation $y=5 R / 2$, noté $\left[Q^{-}, \Omega_{2}^{-}\right]$, compris entre $(\mathcal{H})$ et $(O y)$ par une rotation de centre $\Omega_{2}^{-}$et d'angle $-\pi / 2$. Le pied de la droite oblique se trouvant en $\left(O^{\prime}\right)$ à la distance $3 R / 2$ à gauche de la première graduation sur la figure intermédiaire 17 , se retrouve à présent en $P_{2}$ à la distance $3 R / 2$ à gauche de $(\mathcal{H})$ sur la figure initiale 18. Cela conduit finalement au graphique de la figure 20.

On pourra enfin noter que la droite associée à $g$, qui a pour équation $y=2 x$, est exceptionnellement perpendiculaire à l'une des diagonales du rectangle $(A B C D)$.

\section{VII Épilogue}

Nous terminerons la rédaction de cet article en reproduisant (avec son aimable autorisation) le dernier paragraphe de l'encadré écrit par A. Dufour dans le numéro de Nexus succédant à la publication de l'article de J. Colmer [3] :

En conclusion, je crois important de rappeler ce que j'écrivais dans le $n^{\circ} 42$ de Nexus : ce petit jeu mathématique s'inscrit en marge de l'article de Colmer, mais n'a rien à voir avec son hypothèse de dédoublement des galeries et chambres de la grande pyramide, qui demeure passionnante. Cela n'a pas davantage à voir avec le vrai angle du triangle directeur 
de la pyramide, dont la valeur n'est ni $51^{\circ} 56^{\prime} 26^{\prime \prime}$, ni les $51^{\circ} 51^{\prime} 14^{\prime \prime}$ proposés par Colmer. Quant à l'angle de $51^{\circ} 49^{\prime} 53^{\prime \prime}$ résultant des mesures en coudées rapportées par Matila Ghyka, là aussi un doute subsiste toujours, car les documents anciens font état de plusieurs " coudées » variant de 44,4 cm à 59,2 cm, la coudée royale égyptienne se situant à peu près à michemin entre ces extrêmes. Or des recherches récentes et en cours conduisent à se demander si la pyramide attribuée à Khéops n'est pas en réalité beaucoup plus ancienne, produit d'une civilisation disparue très avancée... Il est temps que l'establishment scientifique accepte de mettre en question ses vieux paradigmes. Affaire à suivre.

Remerciements. L'auteur remercie Jean-François Pioche pour sa contribution à la section III et Frédéric De Ligt pour sa contribution à la sous-section V.1, ainsi que Jean-Claude Carréga pour une lecture attentive de ce travail et ses encouragements. L'auteur exprime sa reconnaissance tout particulièrement envers André Dufour qui est à l'origine de la construction présentée dans la section VI et plus spécifiquement des graphiques référencés des figures 19 et 20 , ainsi que de nombreuses correspondances très fructueuses.

\section{Références}

[1] J.-C. Carréga, Théorie des corps, la règle et le compas, Hermann, Paris, 2001.

[2] J. Colmer, « Des chambres secrètes dans la grande pyramide», Nexus 42 (janvier-février 2006) 1521.

[3] A. Dufour, «Khéops, J. Colmer et la géométrie», Nexus 43 (mars-avril 2006) 78; « erratum » (courrier des lecteurs), Nexus 44 (mai-juin 2006) 78. 